\title{
Determinants of pigeons' choice between certain and probabilistic outcomes
}

\author{
MARCIA L. SPETCH, MICHAEL V. MONDLOCH, and TERRY W. BELKE \\ University of Alberta, Edmonton, Alberta, Canada \\ and \\ ROGER DUNN \\ San Diego State University, San Diego, California
}

\begin{abstract}
Pigeons chose between $50 \%$ and $100 \%$ reinforcement on a discrete-trials concurrent-chains procedure with fixed-ratio 1 initial links and fixed-time terminal links. The $100 \%$ alternative always provided food after a terminal-link delay, whereas the $50 \%$ alternative provided food or blackout equally often after a delay. Additionally, the terminal-link stimuli on the $50 \%$ alternative were correlated with the outcomes in signaled, but not in unsignaled, conditions. The effects of intertrial-interval duration and length of the terminal-link delays on choice of the $50 \%$ alternative were investigated in four experiments. Preference for the $50 \%$ alternative varied with signal condition and duration of the terminal link leading to food, but not with duration of either intertrial interval or the terminal link leading to a blackout. The results are discussed in terms of conditioned-reinforcement effects, Mazur's hyperbolic-decay model, and delay reduction.
\end{abstract}

Choice between certain and uncertain outcomes has proved fertile ground for the study of the combined effects of primary and conditioned reinforcement. In one of the more common procedures used to study choice with probabilistic outcomes in animals (concurrent chains), a delay (terminal link) is imposed between the choice response and an outcome. On one alternative, the outcome is always food ( $100 \%$ reinforcement), whereas on the other alternative, the outcome is sometimes food (e.g., at 50\%) and sometimes blackout. When the choice requirement is minimal (e.g., a single response), and when choice requirements and terminal-link durations for the two alternatives are equal, reinforcement is maximized by exclusive choice of the $100 \%$ reinforcement alternative.

If maximization of food reinforcement were the only determinant of choice in this context, subjects should always choose the $100 \%$ alternative, regardless of terminal-link duration. However, pigeons do not always respond in this fashion (e.g., Dunn \& Spetch, 1990; Kendall, 1974, 1985; Spetch, Belke, Barnet, Dunn, \& Pierce, 1990). Instead, preference depends on the contingencies associated with the $50 \%$ alternative. For example, when the outcomes on the $50 \%$ alternative are not signaled by (i.e., are uncorrelated with) the terminal-link stimuli, pigeons reliably choose the $100 \%$ alternative

This research was supported by a Natural Sciences and Engineering Research Council of Canada research grant awarded to the first author. We thank Ron Kelly, Tim Robinson, and Siew Tan for assistance with the research. Correspondence concerning this article should be addressed to Marcia L. Spetch, Department of Psychology, University of Alberta, Edmonton, Alberta, Canada, T6G 2E9 (e-mail: mspetch@) psych.ualberta.ca).
(Spetch et al., 1990). However, when terminal-link stimuli on the $50 \%$ alternative do signal (i.e., are correlated with) the outcomes, choice allocation moves away from exclusive preference for the $100 \%$ alternative, despite the fact that overall rate of reinforcement diminishes as a consequence. Moreover, as the terminal-link delay increases in signaled procedures, choice of the unreliable (i.e., 50\%) alternative increases (Spetch et al., 1990).

Choice of the $50 \%$ alternative in such procedures is difficult to reconcile with most models of choice behavior. It is clearly inconsistent with any model based on molar maximization of primary reinforcement because choice of the $50 \%$ alternative lowers the overall rate of food reinforcement obtained. For example, optimal foraging models (e.g., Krebs, 1978; Pyke, Pulliam, \& Charnov, 1977) would predict rejection of the $50 \%$ alternative because the energy gain per unit of time spent foraging is lower than for the $100 \%$ alternative. Models based on molecular maximization (Shimp, 1966) or melioration (Herrnstein \& Vaughan, 1980) also have difficulty explaining choice of the $50 \%$ side if only primary reinforcement is considered, because the momentary probability of food and the local rate of food reinforcement are always lower for the $50 \%$ alternative than they are for the $100 \%$ alternative.

Other models specifically formulated to deal with choice for probabilistic reinforcement do not predict choice of the unreliable schedule, either. For example, under some circumstances, risk-sensitive foraging models predict that animals should select unreliable alternatives over reliable ones (e.g., Caraco, 1983), but this prediction applies only to situations in which the unreliable reward is larger than the reliable one. Specifically, such 
risk-prone foraging may be adaptive when choice of the reliable alternative provides insufficient energy and would eventually lead to starvation. In that case, choice of a larger, but uncertain, reward provides a chance to avoid certain starvation. In situations involving equal amounts of reinforcement per instance for the reliable and unreliable alternatives, however, risk-prone behavior would not be adaptive. A model described by Rachlin, Logue, Gibbon, and Frankel (1986), which assumes that probabilistic reinforcers are functionally equivalent to delayed reinforcers, does not predict the observed tendency to choose the $50 \%$ alternative, either. With equal choice requirements and equal terminal-link durations on the two alternatives, the converted probabilities yield a delay on the $50 \%$ side that is double that on the $100 \%$ side. Preference for the $100 \%$ side is therefore always predicted, provided that animals are sensitive to the differences in the delay. Insensitivity to such differences does not provide a plausible account of pigeons' suboptimal choice in signaled procedures because strong preferences for the $100 \%$ alternative occur under the same temporal parameters in unsignaled procedures (e.g., Dunn \& Spetch, 1990; Spetch et al., 1990).

In short, choice of the $50 \%$ alternative in signaled procedures cannot be explained by primary-reinforcement effects alone. The contrast between the signaled and unsignaled results suggests instead a role played by the signals for food (i.e., the conditioned-reinforcing properties of the terminal-link stimuli). Along these lines, Dunn and Spetch (1990) and Spetch et al. (1990) proposed a framework that borrows heavily from Fantino's (1969) delay-reduction hypothesis of conditioned reinforcement. This will be referred to as the "local delayreduction framework," both to distinguish it from the original hypothesis and to indicate that, as yet, it represents only an approach to understanding choice between certain and probabilistic outcomes, not a formalized model. According to this framework, choice between certain and probabilistic outcomes is jointly controlled by delayed primary reinforcement and the effectiveness of the terminal-link stimuli as conditioned reinforcers. As in Fantino's delay-reduction hypothesis, the conditionedreinforcing effectiveness of the terminal-link stimuli is assumed to be governed by the reduction in delay to food signaled by their onset. However, the Dunn and Spetch version adds a relative-validity assumption-namely, that the conditioned reinforcement provided by the onset of a terminal-link stimulus is undermined if it is redundant as a signal for a reduced delay to primary reinforcement. Such redundancy would exist when the 100\% alternative is chosen, but not when the $50 \%$ alternative is chosen, in a concurrent-chains procedure with a fixedratio (FR) 1 choice contingency. Specifically, under these conditions, choice of the $100 \%$ alternative is always followed by reinforcement after a fixed delay, so onset of the $100 \%$ terminal-link stimulus signals little, if any, reduction in that delay. On the other hand, choice of the $50 \%$ alternative is not always followed by food after a fixed delay. Consequently, onset of the food-correlated terminal-link stimulus does uniquely signal a substantial reduction in the delay to reinforcement. Thus, the effectiveness of the terminal-link stimuli as signals for delay reduction is assumed to depend upon the local context of the choice peck. The assumption that local context can provide an important comparator seems consistent with evidence from other procedures (e.g., Barnet, Grahame, \& Miller, 1993).

According to this local delay-reduction framework, choice of the $50 \%$ alternative occurs in the signaled discrete-choice concurrent-chains procedure when (1) control by primary reinforcement (which favors the $100 \%$ alternative) is relatively weak; and (2) conditioned reinforcement favors the $50 \%$ alternative. Control by primary reinforcement is assumed to be weak when there is a substantial delay between choice and the eventual delivery of food. Thus, with 30 -sec terminal links it should be weaker than with 5-sec terminal links (see also Killeen, 1982). Conditioned reinforcement is assumed to favor the $50 \%$ alternative with an FR 1 choice requirement because with this requirement, onset of the $100 \%$ terminal-link stimulus is redundant as a signal for delay reduction, whereas onset of the terminal-link stimulus signaling food (i.e., the $\mathrm{S}+$ ) on the $50 \%$ side is not. In other words, the $\mathrm{S}+$ on the $50 \%$ side should function more effectively as a conditioned reinforcer. One additional assumption is needed to explain the tendency to choose the $50 \%$ side - namely, that onset of the stimulus signaling blackout (the $\mathrm{S}-$ ) does not exert strong punishing effects. This assumption is supported by evidence from other contexts (Dunn, 1990; Williams \& Dunn, 1991).

An important role for conditioned reinforcement has also been implicated in studies of choice between certain and probabilistic outcomes using adjusting-delay procedures (e.g., Mazur, 1989, 1991). In this procedure, pigeons choose between an alternative that provides a probabilistic reinforcer after a fixed delay and one that provides a certain reinforcer after a delay that is adjusted in duration until an indifference point is found. Mazur (1991) found that the indifference point varied dramatically as a function of whether or not the stimulus conditions for the probabilistic alternative on trials leading to food were the same as those on trials leading to no food. Specifically, the comparison delay at the indifference point was much longer when the stimuli on these trials were identical (analogous to the unsignaled procedure described above) than it was when the stimuli that were present on trials leading to food were omitted on trials without food (analogous to the signaled procedure). Mazur accounted for these results in terms of a hyperbolicdecay model (e.g., Mazur, 1989), modified to emphasize conditioned-reinforcement effects (see Mazur, 1991, 1993; Mazur \& Romano, 1992). According to this model, the value of a conditioned reinforcer decreases hyperbolically with increasing delay to the primary reinforcer, but only with delays spent in the presence of the conditioned reinforcer. In the case of probabilistic reinforcement, probability is assumed to function like delay, such 
that as the probability of reinforcement in the presence of an $\mathrm{S}+$ decreases, the functional delay to reinforcement increases. Consequently, the value of that conditioned reinforcer decreases. However, if the stimuli associated with food are not present during the intervals leading to nonreinforcement (as in signaled procedures), these nonreinforced intervals are not a functional part of the delay to the reinforcer. Consequently, the conditionedreinforcing value of the $\mathrm{S}+$ changes little as probability of reinforcement changes.

Mazur's model predicts the large difference between signaled and unsignaled conditions of the discrete-trial concurrent-chains procedure because only in the unsignaled procedure would time spent in the delay ending in blackout lower the value of the stimuli associated with the $50 \%$ alternative. Specifically, in the unsignaled procedure, the average wait for food in the presence of stimuli associated with food is typically twice as long on the $50 \%$ side as it is on the $100 \%$ side, so a strong preference for the $100 \%$ side should occur. In the signaled procedure, however, only one stimulus $(\mathrm{S}+)$ is associated with food on the $50 \%$ alternative, so the delay to food in its presence is equal to the delay to food in the presence of the $100 \%$ terminal-link stimulus. Consequently, the only difference between the alternatives that would affect their value is that, on average, twice as many initiallink periods are required per reinforcer on the $50 \%$ side; choice latency is assumed to be a functional part of the delay to the reinforcer (see Mazur, 1991). When only a single choice peck is required, however, the decrease in value of the $50 \%$ side produced by the extra initial-link time would be relatively small. Thus, little or no systematic preference for the $100 \%$ side would be expected, particularly when terminal-link durations are relatively long.

The present series of experiments further investigated choice with uncertain outcomes in the discrete-trial concurrent-chains procedures. These investigations were designed not to test specific contrasting predictions of the two frameworks outlined above, but rather to provide a broader empirical base of knowledge about choice between certain and probabilistic outcomes. This is important because compared with the vast literature on choice between alternatives involving certain outcomes, the literature on choice with probabilistic outcomes remains quite limited. Of particular interest here was the relationship between preference and temporal parameters such as intertrial-interval (ITI) duration, blackout-stimulus duration, and food-stimulus duration. Within the adjusting-delay procedure, only intervals of time spent in the presence of stimuli associated with food appear to substantially alter the value of a probabilistic alternative. Time spent in the presence of stimuli not associated with food, such as the ITI or signaled delays to blackout, appear to have little or no effect on the value of a probabilistic alternative (Mazur, 1991). By comparison, within our discrete-trial concurrent-chains procedure, preference for a probabilistic alternative is affected by the duration of equal terminal links, although it is not known if this is due to the duration of the food terminal links, the blackout terminal links, or both. Accordingly, in the present studies, we attempted to isolate the particular intervals that influence pigeons' choice of $50 \%$ rather than $100 \%$ reinforcement to determine whether or not results obtained in the discrete-trial concurrent-chains procedure would mirror those obtained in the adjusting-delay procedure.

\section{EXPERIMENT 1}

Mazur $(1989,1991)$ reported that manipulations of the ITI had no consistent effect on pigeons' choice between certain and probabilistic rewards within an adjusting-delay procedure. On the other hand, increases in the ITI have been found to diminish human subjects' tendency to choose uncertain large rewards over certain small ones in a discrete-trials hypothetical gambling procedure (Rachlin et al., 1986). In the discrete-trial concurrent-chains procedure with signaled outcomes, Spetch et al. (1990) found no consistent effect of ITI duration on choice between $100 \%$ and $50 \%$ reinforcement. However, their study manipulated ITI only in the context of short terminal-link durations, in which pigeons displayed preference for the $100 \%$ alternative. The present experiment examined the effect of ITI in the context of longer terminal links to determine whether ITI would affect choice within conditions that yield a higher tendency to choose the $50 \%$ alternative.

\section{Method}

\section{Subjects}

Eight experimentally naive adult pigeons were maintained at approximately $85 \%$ of their free-feeding weights by access to mixed grain obtained during and after experimental sessions. All birds were housed in large individual cages under a 12-h light:dark cycle. Sessions were conducted from 5 to 7 days per week and began from 1 to $7 \mathrm{~h}$ after light onset, at approximately the same time each day for individual birds. Water and grit were available ad lib in the home cages.

\section{Apparatus}

Two three-key chambers and two two-key pigeon chambers were used. Only the side keys of the three-key chambers were used. The pecking keys, for which a force of approximately $0.2 \mathrm{~N}$ was required to operate, were aligned horizontally approximately $20 \mathrm{~cm}$ above the floor. Projectors mounted behind each key were used to project colored fields onto the keys. The houselight was centered above the pecking keys, with the light directed toward the ceiling. A grain feeder was centered below the pecking keys. A lamp located in the feeder was illuminated during food presentations. An exhaust fan provided ventilation and masking noise. Stimulus presentations and recording of data were controlled by a microcomputer located in an adjacent room.

\section{Procedure}

Preliminary training. Each bird received one or more sessions of magazine training followed by several sessions of keypeck training. During keypeck-training sessions, the color that was to serve as the initial-link stimulus was intermittently presented for $8 \mathrm{sec}$ on a side key. Food was presented immedi- 
ately if the bird pecked the illuminated key, or at the end of the 8 -sec period, whichever came first. Trials with each side key occurred equally often in a randomly determined order within each session. Keypeck training continued until each bird pecked on more than $50 \%$ of the trials on each side key for two consecutive sessions.

Signaled discrete-trial concurrent-chains procedure. The procedure used for this experiment is illustrated in Figure 1. Each trial began with onset of the initial link (i.e., choice phase) in which both side keys were illuminated with the same color. A single peck to either key (fixed ratio 1, FR 1) initiated the terminallink schedule by changing the color on that key and rendering the other key dark and inoperative. The terminal link ended after a fixed time (FT) with an outcome (a 4-sec presentation of food or a 4 -sec blackout). The terminal link on the $100 \%$ side always ended with a food outcome, whereas the terminal link on the $50 \%$ side ended with either a food outcome or a blackout, each with a probability of .5 . The $50 \%$ alternative was on the right key in Chambers 1 and 4 and on the left key in Chambers 2 and 3. The stimulus assignments varied across the four chambers. The initiallink stimuli were yellow in Chamber 1 and red in the remaining chambers. The $100 \%$ terminal-link stimulus was orange in Chamber 1, white in Chambers 2 and 3, and yellow in Chamber 4. The $50 \% \mathrm{~S}+$ terminal link was yellow in Chamber 2 and green in the remaining chambers. The $50 \% \mathrm{~S}-$ terminal link was red in Chamber 1, green in Chamber 2, yellow in Chamber 3, and white in Chamber 4. Two birds were assigned to each of the four chambers.

The FT values used for all terminal links (i.e., $\mathrm{X}, \mathrm{Y}$, and $\mathrm{Z}$ in Figure 1) were equal throughout this experiment. During an initial training phase, the FTs were set to $2 \mathrm{sec}$ for the first two sessions, to $15 \mathrm{sec}$ for the next two sessions, and to $30 \mathrm{sec}$ for the final two sessions. The ITI was $15 \mathrm{sec}$ for all birds during these 6 training sessions. During the subsequent experimental phases, the terminallink FTs remained constant at $30 \mathrm{sec}$. During the first condition, the ITI was $0 \mathrm{sec}$ for four birds and $30 \mathrm{sec}$ for the other four. The ITI conditions alternated over the next three conditions, so that each bird received two determinations with a 0 -sec ITI and two with a 30 -sec ITI.

Each session began with four warm-up trials which provided forced choice to one of the alternatives. On two of these trials, only the initial link for the $100 \%$ side was available. On the remaining two trials, only the initial link for the $50 \%$ side was available; one of these trials led to the food terminal link and the other led to the blackout terminal link. The order of the four forced-choice trials was randomly determined for each session. The remainder of the session consisted of free-choice trials with both initial links available. Sessions lasted until each bird had obtained 60 reinforcers from the free-choice trials, or for a maximum of $60 \mathrm{~min}$. Data from the warmup trials are not presented and were not included in any analyses.

For each bird, a choice proportion was calculated for each session (initial-link pecks to the $50 \%$ side/initial-link pecks to both sides). Values above .5 indicate preference for the $50 \%$ alternative and values below .5 indicate preference for the $100 \%$ alternative. Each condition remained in effect for a minimum of 15 sessions and until the bird's choice proportions were stable according to the following criteria: the mean choice proportions for three consecutive three-session blocks (1) did not differ by more than .05; and (2) showed neither an increasing nor a decreasing trend. All data reported are averaged over the nine sessions that satisfied these stability criteria. For all statistical tests, the criterion for rejection of the null hypothesis was set to $p<.05$.

\section{Results}

Figure 2 shows the mean of the eight birds' choice proportions for the $50 \%$ alternative during the first and second series of ITI conditions. As can be seen, ITI du-

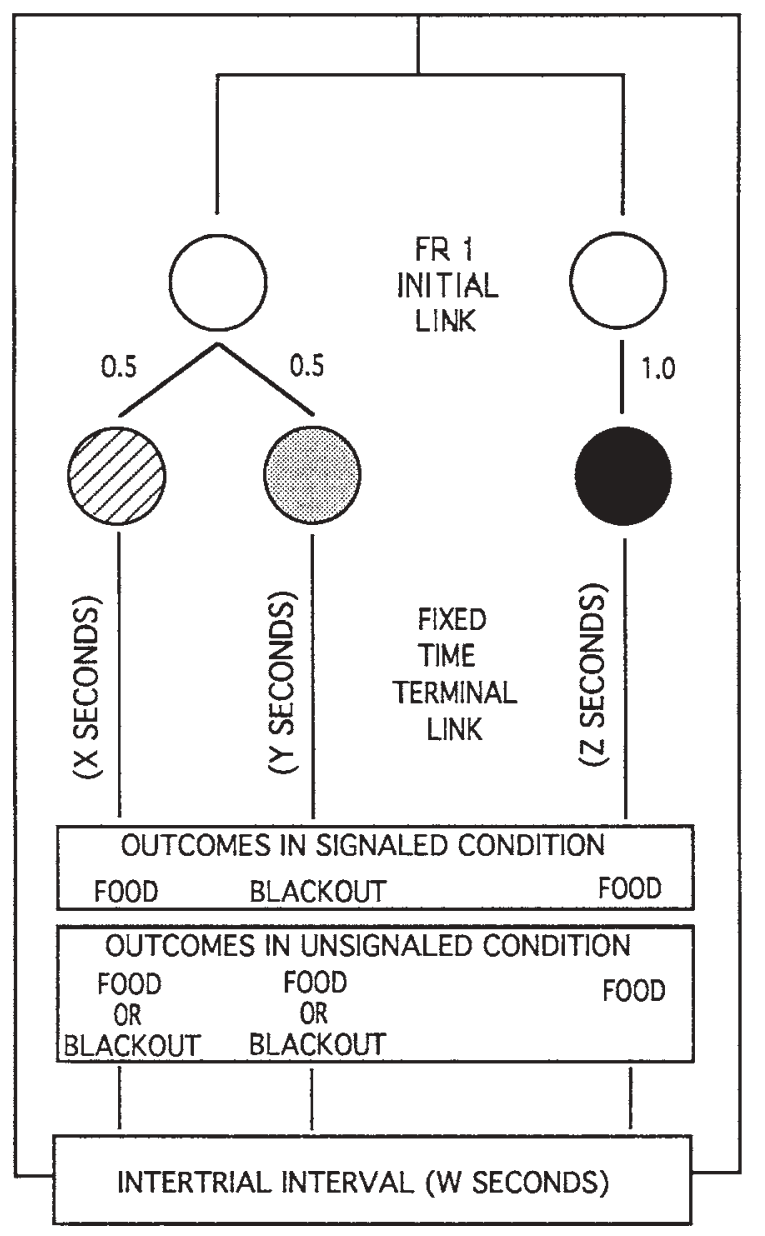

Figure 1. Diagram of the concurrent-chains procedure used in Experiments 1,2 , and 3 . On the $50 \%$ alternative (left), one of two differently colored keylights, each with a probability of .5 , was presented during each terminal-link delay. In signaled conditions, one of the colors was always followed by food and the other was always followed by blackout. In unsignaled conditions, both terminal-link stimuli are followed equally often by food and blackout. The $100 \%$ alternative (right) provides only one terminal-link stimulus and is always followed by food. Except for one condition in Experiment 3, the duration of the $100 \%$ terminal link $(\mathrm{Z})$ and the $50 \%$ terminal link leading to food (X in signaled procedure; $X$ and $Y$ in unsignaled procedure) was $30 \mathrm{sec}$, but the duration of the intertrial interval $(W)$ and the duration of the $\mathbf{5 0 \%}$ terminal link leading to blackout ( $\mathrm{Y}$ in signaled conditions; $\mathrm{X}$ and $Y$ in unsignaled conditions) varied. See text for details.

ration had little effect upon pigeons' tendency to choose the $50 \%$ alternative. A two-way repeated measures analysis of variance (ANOVA) revealed no significant effect of either ITI duration or series $[F(1,7)=0.02$ and $F(1,7)=1.02$, respectively $]$, and no significant interaction $[F(1,7)=1.09]$.

ITI duration did, however, affect terminal-link response rates. Collapsed across the two test series, terminal-link response rates on the $100 \%$ side were significantly higher when the ITI was $30 \mathrm{sec}(M=17.7)$ than when it was $0 \sec [M=14.3 ; t(7)=3.11]$. Response 
rates to the $50 \% \mathrm{~S}+$ terminal link were also significantly higher when the ITI was $30 \mathrm{sec}(M=22.5)$ than they were when it was $0 \sec [M=14.9 ; t(7)=2.36]$, whereas response rates to the $50 \% \mathrm{~S}$ - terminal link did not significantly differ between the two ITI conditions [ 0 -sec ITI, $M=0.4 ; 30$-sec ITI, $M=0.6 ; t(7)=1.88]$.

Under both ITI conditions, the birds made more responses to the $50 \% \mathrm{~S}+$ terminal link than they did to the $100 \%$ terminal-link stimulus. Because of considerable variability in absolute response rates, this comparison between response rates to the $100 \%$ stimulus and to the $50 \% \mathrm{~S}+$ was analyzed by computing a terminal-link response-rate ratio for each bird, as follows: rate for the $50 \% \mathrm{~S}+$ divided by the sum of the rates for the $50 \% \mathrm{~S}+$ and the $100 \%$ stimulus. Scores above .5 indicate higher response rates for the $50 \% \mathrm{~S}+$ and scores below .5 indicate higher response rates for the $100 \%$ stimulus. These ratios were significantly above .5 under both ITI conditions [30-sec ITI, $M=.659, t(7)=3.28 ; 0-\mathrm{sec}$ ITI, $M=.706, t(7)=2.99$ ], and they did not differ across ITI condition $[t(7)=1.11]$. However, there was no significant correlation between these relative response-rate scores and initial-link choice proportions in either ITI condition [0-sec ITI, $r=-.083, t(6)=0.203$; $30-$ sec ITI, $r=.092, t(6)=0.225]$.

\section{Discussion}

These results showed that choice of the $50 \%$ side was not affected by the duration of the ITI. This is interesting because increasing the ITI decreases the overall rate of reinforcement available on the $50 \%$ alternative more

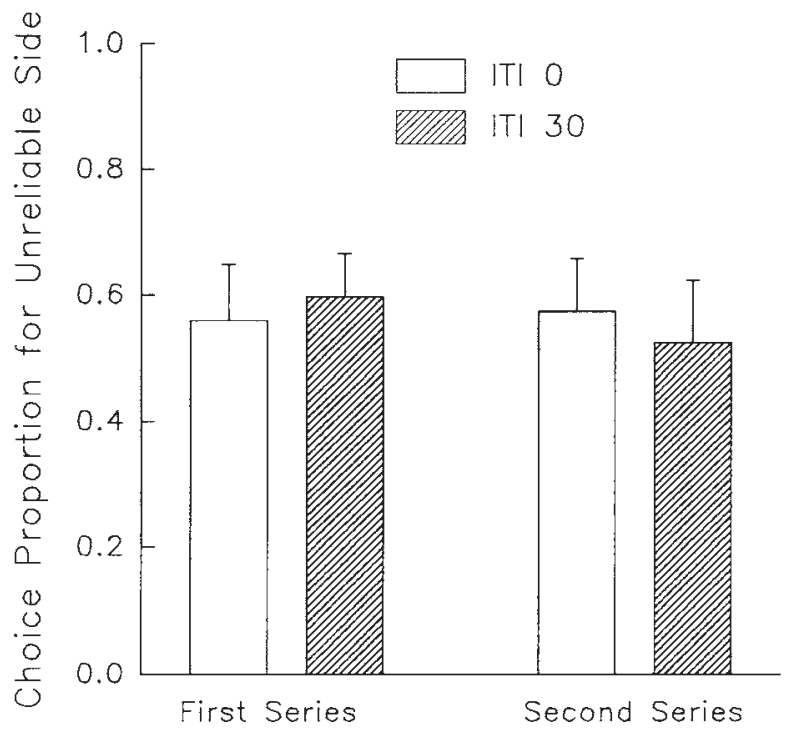

Figure 2. Mean $( \pm S E M)$ choice proportions of the unreliable $(50 \%)$ alternative during the first and second series of conditions with intertrial intervals (ITI) of $\mathbf{0}$ and $30 \mathrm{sec}$ in signaled conditions of Experiment 1. than it decreases the overall rate of reinforcement available on the $100 \%$ side. These results are consistent with previous results obtained by Spetch et al. (1990) and Mazur (1989), in suggesting that the relative value of an alternative leading to probabilistic reward is not altered by the duration of the ITI when the stimulus conditions during the ITI differ from those present during the trial. At the same time, the duration of the ITI was not completely without effect on behavior, because rate of responding to the terminal-link stimuli associated with food on both sides was higher when the ITI was long than it was when the ITI was short. This result is consistent with findings from classical conditioning studies showing that rates of elicited responding to a CS for food increase as a function of ITI (e.g., Terrace, Gibbon, Farrell, \& Baldock, 1975). The finding that the ITI affected absolute rates of responding to both food terminal links, but failed to alter either the relative rates to the $50 \%$ versus the $100 \%$ food terminal links or the choice proportions for the $50 \%$ alternative, suggests that the ITI may have affected equally the value of both the $100 \%$ terminal-link stimulus and the $50 \% \mathrm{~S}+$, hence leaving their relative values unchanged. Another interpretation of the increased terminal-link rates with longer ITIs is that when the terminal links preceded a signaled period of extinction (the ITI), those links produced an anticipatory contrast effect (Williams, 1979, 1992a). It is important to note that increased response rates in the presence of a stimulus followed by a period of extinction are not necessarily indicative of the strength of that stimulus as a conditioned reinforcer (Williams, 1992b).

\section{EXPERIMENT 2}

In percentage reinforcement procedures that are signaled, but not in those that are unsignaled, a majority of subjects show a preference for the $50 \%$ alternative (e.g., Spetch et al., 1990). Thus, differentiating the delays leading to blackout (with an $\mathrm{S}-$ ) from those leading to food (with an $\mathrm{S}+$ ) alters the value of the $50 \%$ alternative. As mentioned above, this result also suggests that the presentation of an $\mathrm{S}$ - signal does not punish choice responses. Beyond this, however, it is not clear how the $\mathrm{S}$ - terminal link affects choice. Previous work has shown that the tendency to select the $50 \%$ alternative is more robust when all terminal-link durations are long (e.g., $30 \mathrm{sec}$ ) than it is when they are short (e.g., 5 or $10 \mathrm{sec}$ ), but because these manipulations varied the duration both of stimuli leading to food and of those leading to blackout, the precise locus of this effect cannot be isolated. Experiment 2 therefore manipulated just the duration of the terminal link leading to blackout.

\section{Method}

\section{Subjects and Apparatus}

The subjects and apparatus were the same as those described in Experiment 1 . This experiment began directly after completion of Experiment 1. 


\section{Procedure}

The basic discrete-trial concurrent-chains procedure was the same as that used in Experiment 1, except that the duration of the terminal link leading to blackout (BOTL) on the $50 \%$ side was set to either $5 \mathrm{sec}$ or $55 \mathrm{sec}$. The duration of the terminal link leading to food on the $50 \%$ side and the duration of the terminal link on the $100 \%$ side were both $30 \mathrm{sec}$ throughout. Thus, in the procedure illustrated in Figure 1 for signaled outcomes, $\mathrm{X}$ and $\mathrm{Z}$ were both $30 \mathrm{sec}$ and $\mathrm{Y}$ was either $5 \mathrm{sec}$ or $55 \mathrm{sec}$. Four birds (one from each chamber) were initially assigned to the 5 -sec condition, and the remaining four were assigned to the $55-\mathrm{sec}$ condition. Thereafter, the conditions alternated, so that each bird received two tests with each duration. The ITI was $0 \mathrm{sec}$ throughout this experiment. The criteria for completion of conditions were the same as those used in Experiment 1.

\section{Results}

Figure 3 shows the mean choice proportions for the $50 \%$ side during the 5 -sec and the 55 -sec conditions in each of the two tests. As can be seen, the duration of the BOTL had little effect upon pigeons' tendency to choose the $50 \%$ alternative. A two-way repeated measures ANOVA revealed no significant effect of BOTL duration or series $[F(1,7)=0.84$ and $F(1,7)=2.82$, respectively], and no significant interaction $[F(1,7)=0.33]$.

BOTL duration also had no significant effect on absolute rate of responding to either the $100 \%$ terminal-link stimulus $[t(7)=1.15]$ or the $50 \% \mathrm{~S}+$ terminal link $[t(7)=$ 0.89]. Under both BOTL conditions, the birds made more responses to the $50 \% \mathrm{~S}+$ terminal link than they did to the $100 \%$ terminal-link stimulus. Averaged across the two test series, relative rates of responding to the $\mathrm{S}+$ rather than to the 100\% terminal link (calculated as described in Experiment 1 ) were significantly above .5 during both the 5 -sec and the 55 -sec conditions $[M=.848, t(7)=6.39$ and $M=.829, t(7)=5.60$, respectively], and these did not differ from each other $[t(7)=0.72]$. These relative terminallink rates were not correlated with initial-link choice proportions during either condition [ $5 \mathrm{sec}, r=.053, t(6)=$ $0.13 ; 55 \mathrm{sec}, r=.098, t(6)=0.24]$.

\section{Discussion}

These results show that choice proportions for the $50 \%$ side are insensitive to variance in overall rate of reinforcement produced by manipulating the duration of the $\mathrm{S}-$. The rate of food reinforcement available on the $50 \%$ side is only slightly lower than that available on the $100 \%$ side when the $\mathrm{S}-$ terminal link is $5 \mathrm{sec}$, but it is substantially lower than that on the $100 \%$ side when the $\mathrm{S}-$ is $55 \mathrm{sec}$. Yet mean choice of the $50 \%$ side was slightly above .5 in both conditions. Thus, time spent in the presence of a stimulus not associated with food does not significantly alter the value of the $50 \%$ side, even when it substantially alters the overall rate of primary reinforcement provided by choice of that side.

\section{EXPERIMENT 3}

This experiment was designed to replicate and extend Experiment 2 by varying the duration of the BOTL

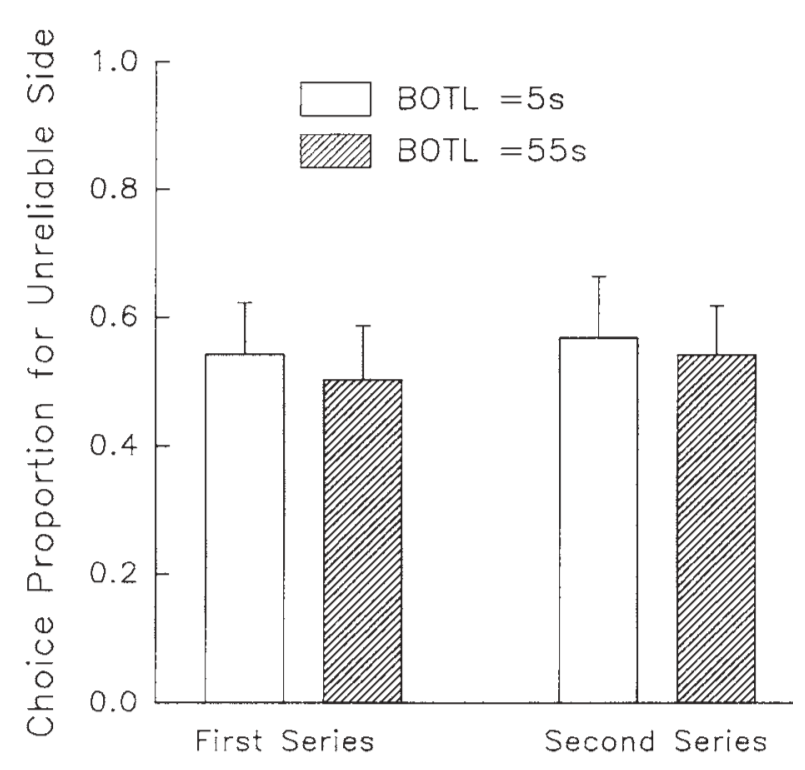

Figure 3. Mean $( \pm S E M)$ choice proportions of the unreliable $(50 \%)$ alternative during the first and second series of conditions with blackout terminal-link (BOTL) durations of 5 or $55 \mathrm{sec}$ in signaled conditions of Experiment 2.

within both signaled and unsignaled procedures. In both procedures, increasing the duration of the BOTL reduces the rate of primary reinforcement provided by the $50 \%$ alternative. Moreover, in unsignaled conditions, both terminal-link stimuli on the $50 \%$ side occur equally often prior to food and blackout outcomes. Consequently, increasing the duration of the BOTL increases the average delay to food associated with each terminal-link stimulus on the $50 \%$ alternative. Therefore, on the basis of either primary-reinforcement or conditionedreinforcement effects, increases in BOTL duration would be expected to reduce choice of the $50 \%$ alternative in unsignaled conditions. This study also included a replication of conditions reported in Spetch et al. (1990), in which all terminal links were short (5 sec ) or long $(30 \mathrm{sec})$ in signaled and unsignaled procedures.

\section{Method}

\section{Subjects}

The subjects were eight adult Silver King pigeons. All had previously been trained in spatial search and/or delayed matching-tosample tasks, but none had been exposed previously to a concurrent-chains procedure. The birds were housed and maintained as described in Experiment 1.

\section{Apparatus}

The apparatus was the same as that described in Experiment 1. Two pigeons were assigned to each of the four chambers.

\section{Procedure}

Each bird received two or more sessions of keypeck training, as described in Experiment 1, followed by forced-choice training on either a signaled or an unsignaled discrete-trial concurrent-chains percentage reinforcement procedure. The signaled procedure was 
the same as that described in Experiment 1. The unsignaled procedure was identical to the signaled one except that each of the two terminal-link stimuli on the $50 \%$ side ended sometimes with food and sometimes with blackout $(p=.5)$, as indicated in Figure 1 (outcomes in unsignaled condition). All terminal-link durations were $2 \mathrm{sec}$ during the first two sessions, $15 \mathrm{sec}$ during the next two sessions, and $30 \mathrm{sec}$ during a third pair of sessions. The BOTL $(\mathrm{S}-$ ) was then set to $5 \mathrm{sec}$ for two birds on the signaled procedure and two birds on the unsignaled procedure, and to $55 \mathrm{sec}$ for the remaining four birds. Each bird received two additional sessions of forced choice and then was moved to free-choice sessions.

All free-choice sessions began with eight forced-choice warmup trials, which were not included in the data summaries or analyses. Four of the warm-up trials provided access to the $100 \%$ terminal link, and four to the $50 \%$ terminal link. Two of these $50 \%$ terminal-link entries ended in food and two in blackout. Sessions lasted until the bird obtained 60 reinforcers on free-choice trials, or for a maximum of $60 \mathrm{~min}$.

Each bird was exposed to three phases, each of which included four conditions. Phases 1 and 3 were identical. The four conditions in each of these two phases differed in the duration of the BOTL $(5 \mathrm{sec}$ or $55 \mathrm{sec}$ ) and in signal condition (signaled vs. unsignaled outcomes). During each of these conditions, all terminal links that ended in food were $30 \mathrm{sec}$ in duration. The order of exposure to conditions varied across birds. Phase 2 entailed variation in the duration of equal terminal links. During the first two Phase 2 conditions, all terminal links were $30 \mathrm{sec}$ in duration, and during the last two Phase 2 conditions, all terminal links were $5 \mathrm{sec}$ in duration. At each duration, the outcomes were signaled for one condition and were unsignaled for the other condition.

To ensure exposure to the new contingencies, all conditions began with two training sessions in which all trials entailed a forced choice. Following this forced-choice exposure, each condition then remained in effect for a minimum of 15 free-choice sessions and until the bird satisfied the stability criteria described in Experiment 1.

\section{Results}

Figure 4 shows the mean choice proportions of the eight birds averaged over the two phases of the 5- and 55 -sec BOTL conditions in the signaled and unsignaled procedures. Consistent with the results obtained in Experiment 2, BOTL duration did not substantially affect choice proportions within the signaled procedure $[t(7)=$ $1.05, p>.1]$. Consistent with our a priori expectations, choice of the $50 \%$ side in unsignaled procedures was higher when the $\mathrm{S}-$ terminal link was $5 \mathrm{sec}$ than when it was $55 \mathrm{sec}$, although this effect was not particularly robust $[t(7)=2.03, p<.05$, one-tailed]. Choice of the $50 \%$ side was also somewhat higher under signaled conditions than it was under unsignaled conditions. Because of high variability, however, the only significant effect revealed by a two-way ANOVA (signal condition $\times$ BOTL duration) was a main effect of BOTL duration $[F(1,7)=6.27]$.

Figure 5 shows the mean choice proportion of the eight birds during the four conditions of Phase 2 in which all terminal links were either $30 \mathrm{sec}$ or $5 \mathrm{sec}$. Consistent with previous results, choice proportions for the $50 \%$ alternative were markedly higher in the signaled procedure than they were in the unsignaled procedure. In addition, a large effect of terminal-link duration was apparent. A two-way ANOVA on these data revealed sig-

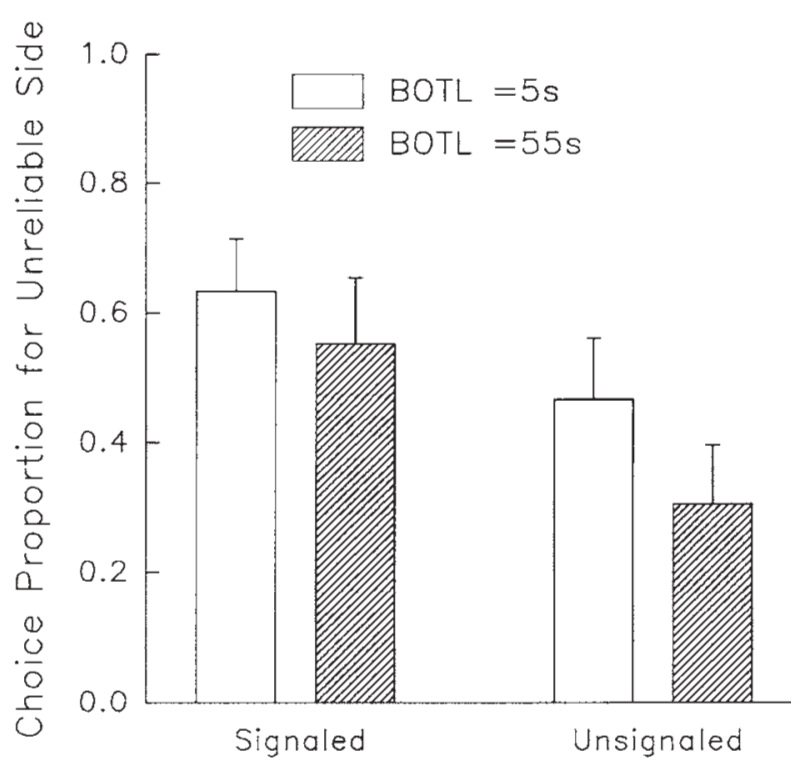

Figure 4. Mean $( \pm S E M)$ choice proportions of the unreliable $(50 \%)$ alternative during signaled and unsignaled conditions with blackout terminal-link (BOTL) durations of 5 or $55 \mathrm{sec}$ in Experiment 3.

nificant effects of both signal condition and terminallink duration $[F(1,7)=7.61$ and $F(1,7)=13.79$, respectively], as well as a significant interaction between signal condition and terminal-link duration $[F(1,7)=$ 9.25].

Terminal-link response rates were not analyzed in this experiment because one or more of the birds displayed exclusive choice of an alternative in several of the conditions, so there was an insufficient number of entries into the terminal link of the other alternative.

\section{Discussion}

In general, the effects of varying the duration of the BOTL were consistent with expectations. Under signaled conditions, preferences were not significantly affected by this manipulation. By contrast, under unsignaled conditions, choice of the $50 \%$ alternative declined as the BOTL duration was increased, although the magnitude of this effect was not large. A different pattern of results was observed when the duration of equal terminal-link durations on both alternatives was varied. When the time to both types of outcome was increased, choice of the $50 \%$ alternative increased under both signaled and unsignaled conditions.

\section{EXPERIMENT 4}

The results of Experiments 1-3 suggest that time spent in intervals not associated with food (the ITI, and the $\mathrm{S}-$ terminal link in signaled procedures) has little effect upon choice. In contrast, choice is consistently affected by manipulation of equal-duration terminal links. 


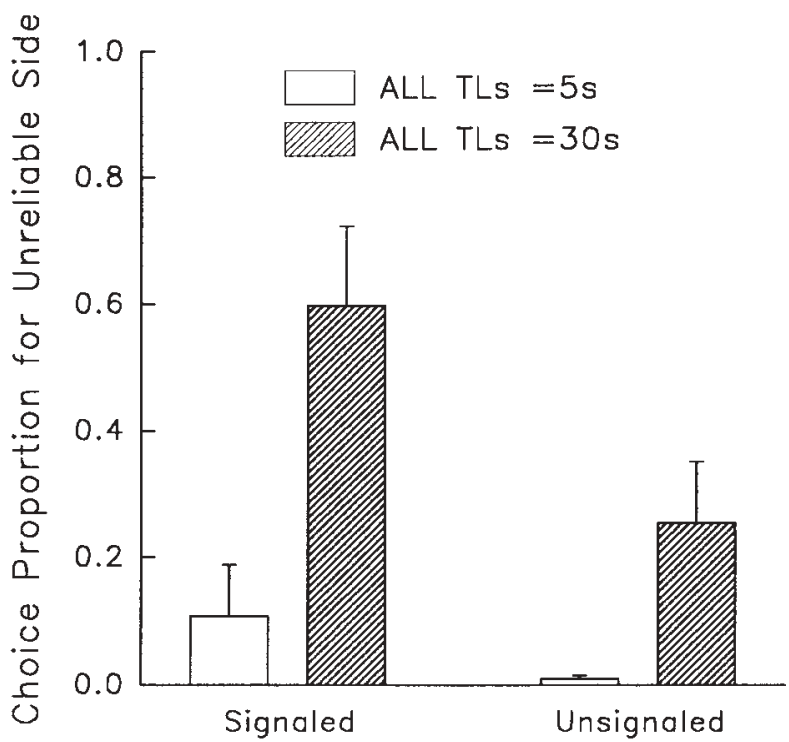

Figure 5. Mean $( \pm S E M)$ choice proportions of the unreliable $(50 \%)$ alternative during signaled and unsignaled conditions with all terminal-link durations equal at 5 or 30 sec in Experiment 3.

Manipulation of terminal-link duration also alters the overall rates of primary reinforcement provided by each alternative. Experiment 4 investigated the effects of changing both the food and blackout terminal-link durations on the $50 \%$ alternative while holding the overall rate of food provided by this alternative constant, and while maintaining an equal average time to an outcome for the $50 \%$ and $100 \%$ alternatives.

\section{Method}

Subjects and Apparatus

The subjects were the eight pigeons from Experiment 2 and four experimentally naive pigeons, all maintained and housed as described in Experiment 1. The apparatus was the same as that described in Experiment 1.

\section{Procedure}

The four naive pigeons were first given magazine and keypeck training as described in Experiment 1. They then received six forced-choice training sessions on the discrete-trial concurrentchains procedure. All terminal links were $2 \mathrm{sec}$ for the first two of these sessions, $15 \mathrm{sec}$ for the next two sessions, and $30 \mathrm{sec}$ for the final two sessions. The procedure used for the remainder of this experiment was similar to that used in the first three experiments, except that the terminal-link durations varied for both alternatives as shown in Figure 6.

All pigeons were exposed, in each of two test series, to four experimental conditions that differed in the terminal-link durations on the $50 \%$ alternative and in signal condition (see Figure 6). In all conditions, the $100 \%$ alternative provided food after $5 \mathrm{sec}$ on $50 \%$ of the trials and after $55 \mathrm{sec}$ on the remaining trials $\left(\mathrm{Z}\right.$ and $\mathrm{Z}^{\prime}$ in Figure 6), so that food was presented after $30 \mathrm{sec}$ on average. The same terminal-link stimulus was present in both cases (i.e., terminal-link duration on the $100 \%$ side was not signaled). In F5/BO55 conditions, half of the trials on the $50 \%$ alternative ended in food after $5 \mathrm{sec}$ and the remaining trials ended in black- out after $55 \mathrm{sec}$. In F55/BO5 conditions, half of the trials on the $50 \%$ alternative ended in food after $55 \mathrm{sec}$ and the remaining trials ended in blackout after $5 \mathrm{sec}$. In signaled conditions, one stimulus always led to food and one stimulus always led to blackout. Thus, both the nature of the outcome (food or blackout) and the duration of the terminal links ( $5 \mathrm{sec}$ or $55 \mathrm{sec}$ ) were signaled by the terminal-link stimuli. In unsignaled conditions, the two terminal-link stimuli equally often led to food or blackout. Thus, the terminal-link stimulus was not correlated with either the duration of

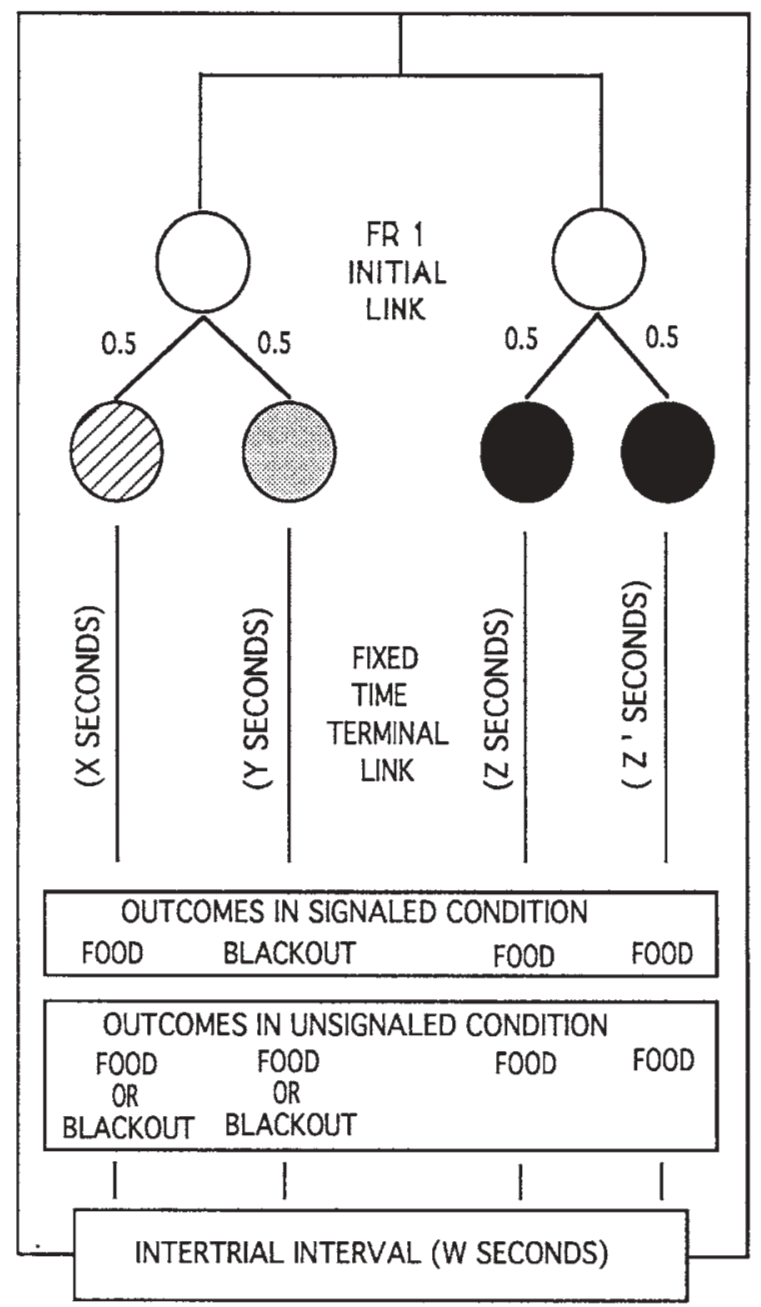

Figure 6. Diagram of the concurrent-chains procedure used in Experiment 4 . On the $50 \%$ alternative (left), one of two differently colored keylights, each with a probability of .5 , was presented during each terminal-link delay. In signaled conditions, one of the colors was always followed by food and the other was always followed by blackout. In unsignaled conditions, both terminal-link stimuli were followed equally often by food and by blackout. The $100 \%$ alternative (right) provides only one terminal-link stimulus and was always followed by food. The $\mathbf{1 0 0} \%$ terminal-link duration was $5 \sec (Z)$ or $55 \sec \left(Z^{\prime}\right)$, each with equal probability. The intertrial interval duration $(W)$ was 0 sec. On the $50 \%$ side, in one condition, the terminal links leading to blackout ( $Y$ in signaled conditions; $X$ and $Y$ in unsignaled conditions) were $5 \mathrm{sec}$ and those leading to food ( $X$ in signaled conditions; $X$ and $Y$ in unsignaled conditions) were $55 \mathrm{sec}$; the reverse was true in the other condition. See text for details. 
the terminal link or the nature of the outcome. It should be noted that the overall rate of primary reinforcement is not affected by these manipulations, and is approximately twice as high on the $100 \%$ side as it is on the $50 \%$ side in all conditions. Moreover, during F5/BO55 conditions, short (5-sec) delays to food occur equally often (half of the time) on both the $50 \%$ and $100 \%$ alternatives; the only difference is that primary reinforcement is provided after the long delay $(55 \mathrm{sec})$ on the $100 \%$ side, whereas blackout is provided after the long delay on the $50 \%$ side. During the F55/BO5 conditions, only the $100 \%$ side provides food after a short delay. The stimulus assignments for this experiment were the same as those described in Experiment 1. Two experienced birds and one naive bird were assigned to each chamber.

The order of exposure to conditions varied across birds, with three birds starting in each of the four conditions. During the first test series, transition between conditions was not preceded by forced-choice sessions, and free-choice sessions began with only one forced-choice warm-up for each outcome on each alternative. The results from the first test series indicated that exclusive preference that developed in one condition tended to carry over into subsequent conditions, suggesting that the four warm-up trials may not have provided sufficient exposure to the new contingencies following a change in conditions. Accordingly, in the second test series, exposure to each of the four conditions began with two forced-choice sessions, and all free-choice sessions started with two warm-up trials for each outcome on each alternative. This additional forced exposure to the contingencies appeared to reduce carry-over effects; consequently, only the results of Test Series 2 are reported.

Each condition remained in effect for a minimum of 15 sessions and until the stability criteria, described in Experiment 1, were satisfied.

\section{Results}

Mean choice proportions from the four conditions are shown in Figure 7. The relative durations of the terminal links leading to food or blackout on the $50 \%$ side had a substantial effect on choice behavior, with much higher choice proportions for the $50 \%$ side when the food terminal link was $5 \mathrm{sec}$ than when it was $55 \mathrm{sec}$. Additionally, this effect was considerably larger in the signaled than in the unsignaled procedure. A two-way ANOVA revealed significant effects of signal condition and relative duration of the food terminal link $[F(1,11)=$ 16. 05 and $F(1,11)=183.83$, respectively], as well as a significant interaction between signal condition and relative food terminal-link duration $[F(1,11)=16.28]$.

Terminal-link response rates were not analyzed in this experiment because the birds frequently showed exclusive preference for one alternative.

\section{Discussion}

Choice proportions for the $50 \%$ alternative were substantially greater when the food-correlated terminal link was $5 \mathrm{sec}$ than when it was $55 \mathrm{sec}$. When the outcomes were signaled, there was a complete reversal of preference with these two terminal-link durations. When the outcomes were unsignaled, the choice proportions for the $50 \%$ alternative increased from near-zero levels to slightly above .5. It is important to note that the relative rate of primary reinforcement on the $50 \%$ alternative did not differ across conditions; the $50 \%$ alternative pro-

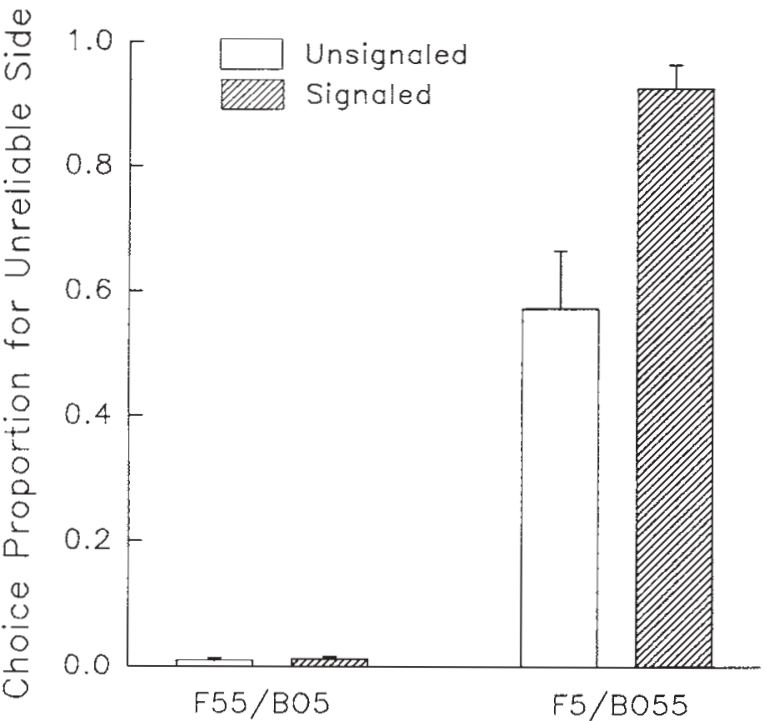

Figure 7. Mean $( \pm S E M)$ choice proportions of the unreliable $(50 \%)$ alternative during signaled and unsignaled conditions when the $\mathbf{5 0} \%$ alternative provided food after $5 \mathrm{sec}$ or blackout after $\mathbf{5 5} \mathrm{sec}$ (F5/BO55), and also when the 50\% alternative provided food after 55 sec or blackout after $5 \mathrm{sec}$ (F55/BO5), in Experiment 4.

vided reinforcement half as often as the $100 \%$ alternative did. Thus, preference was determined by the delay to primary reinforcement and by the stimulus contingencies during the delay. The efficacy of the stimulus contingencies in these results parallels that found in direct comparisons of signaled and unsignaled delays to reinforcement. For example, Hursh and Fantino (1974) compared two alternatives that both provided long and short terminal links. All terminal links ended in food and the terminal-link durations were equal on the two alternatives. They found preference for signaled over unsignaled delays to food. The principal difference between their procedure and the signaled conditions in the present procedure is that in the latter, one of the two terminal links did not end in food. When that terminal link was the longer of the two (i.e., $55 \mathrm{sec}$ ), the signal contingency outweighed the effect of reinforcement after a long delay.

\section{GENERAL DISCUSSION}

The present series of experiments contributed the following observations to our understanding of choice between probabilistic and certain outcomes in a discretetrial concurrent-chains procedure. First, choice proportions for the probabilistic outcome did not vary with ITI duration. Although choice was not affected, terminallink response rates were higher overall when the ITI was longer. Furthermore, response rates in the presence of the food stimulus on the $50 \%$ side were generally higher than they were on the $100 \%$ side, although this differ- 
ence was not correlated with choice proportions. Second, while choice proportions did not vary with the duration of the stimulus leading to blackout on the $50 \%$ side under signaled conditions, they did vary under unsignaled conditions. Third, choice proportions varied with the relative durations of the stimulus associated with food and the stimulus associated with blackout on the $50 \%$ side under conditions in which the average time to an outcome was equivalent on the $100 \%$ and $50 \%$ alternatives. Finally, we replicated the previously reported finding (Spetch et al., 1990) that, when all terminal-link durations are equal, choice proportions for the $50 \%$ side are substantially affected by the duration of the terminal links.

The first finding - that choice did not vary with ITI duration - is consistent with previous findings by Mazur (1989) using the adjusting-delay procedure. It also replicates results by Spetch et al. (1990) and extends those results by showing that ITI had no effect on choice proportions, even under conditions in which pigeons frequently chose the $50 \%$ alternative. The significance of this is that the average time from the choice response to the delivery of food varies with the duration of the ITI on the $50 \%$ alternative but not on the $100 \%$ alternative. Therefore, choice of the $50 \%$ alternative decreases the rate of primary reinforcement to a greater extent when the ITI is long. According to Rachlin et al. (1986), longer ITIs should therefore weaken the value of the probabilistic alternative. Nevertheless, our finding, like those of Mazur (1989), suggests that the signaled period of time between the presentation of an outcome and the next opportunity to choose between outcomes is not functionally related to preference.

Although ITI duration did not affect preference, terminal-link response rates did vary with ITI duration. That is, the subjects responded at higher rates when opportunities to choose were less frequent. This enhancement of responding to stimuli associated with food when ITI is lengthened is consistent with trial-spacing effects on autoshaped response rates (e.g., Terrace et al., 1975). The effect of ITI duration on absolute response rates during food-associated stimuli, in the context of no effect on either preference between the alternatives or relative terminal-link response rates, suggests that lengthening the ITI may have equally enhanced the value of all periods of time associated with food. One possibility is that the $30-\mathrm{sec}$ delay to food associated with the positive terminal-link stimuli was perceptually shorter in the context of 30 -sec ITIs than it was in the context of 0-sec ITIs (see Spetch \& Rusak, 1989, 1992). Another possibility is that responding in the terminal link may have been enhanced by an anticipatory contrast effect maintained by the subsequent period of extinction in the 30-sec ITI (Williams, 1979, 1992a).

The lack of correlation between initial-link choice proportions and relative rates of responding during the $100 \%$ and $50 \%$ food terminal links also suggests that these are not equivalent indicators of the value of the $50 \%$ side. Whereas relative response rates reliably indi- cated higher value of the $50 \%$ food terminal link over the $100 \%$ food terminal link, choice proportions were more variable and suggested that the $50 \%$ side was only slightly more valuable on average than the $100 \%$ side. It may be that choice proportions reflect the joint contribution of the lower rate of primary reinforcement and the higher value of the $50 \%$ food terminal link, whereas rate of responding during the terminal links leading to food reflects only the latter.

Dunn and Spetch (1990) and Spetch et al. (1990) noted that results from the percentage reinforcement procedure were analogous to findings from serial autoshaping studies, in which responding to a stimulus that had an intermittent relationship to the occurrence of a stimulus associated with food was higher than responding to a stimulus that was always followed by a stimulus associated with food (Collins \& Pearce, 1985; Collins, Young, Davies, \& Pearce, 1983). However, Pearce and Collins (1987) used choice probes to test preference between stimuli that were intermittently or perfectly predictive of a stimulus associated with food, and found that their measures of preference did not mirror the difference in response rates found in serial autoshaping. Specifically, the proportion of responses occurring to the stimulus always followed by a stimulus associated with food was higher in the choice test, and the first response on the probe trials was to this stimulus $62 \%$ of the time. Thus, response rates during single successive presentations of the alternatives and preference during simultaneous presentations of the alternatives were dissociated (Pearce \& Collins, 1987). This dissociation was also found in a recent study by Fantino and Case (1993). These results may be consistent with the present finding that there was no relationship between initiallink preference and relative response rates in the terminal links associated with food (see also Farley, 1980; Williams, 1992b).

The second finding was that choice of the $50 \%$ side did not vary significantly with the duration of a signaled terminal link leading to blackout (BOTL), but that it did vary somewhat with the duration of unsignaled BOTLs. In only the latter case were stimuli that were present during the manipulated terminal link also present on some of the trials that ended in food. Although the manipulations and procedures are somewhat different, these results are consistent with results obtained within the adjustingdelay procedures, suggesting that nonreinforced periods reduce the value of a probabilistic alternative more if they are spent in the presence of the signal for food (Mazur \& Romano, 1992). Mazur's hyperbolic-decay model accounts for these findings because it assumes that the value of an outcome declines hyperbolically with delay, but only in the presence of stimuli associated with that outcome. Therefore, the duration of a signaled blackout period on the $50 \%$ alternative would not affect the value of the $50 \%$ alternative and should not affect preference. In the unsignaled procedure, however, BOTLs are spent in the presence of the stimuli associated with food. Consequently, lengthening the BOTLs increases 
the delay to food, and thereby decreases the value of these stimuli.

These results may also be accommodated within the local delay-reduction framework. According to this approach, choice between the $100 \%$ and the $50 \%$ alternatives depends on both primary-reinforcement and conditioned-reinforcement effects. With FR 1 initial links, the $100 \%$ alternative provides the higher rate of primary reinforcement but little, if any, conditioned reinforcement because onset of the terminal-link stimulus signals little reduction in delay over that already signaled by the choice peck. Although the 50\% alternative provides a lower rate of primary reinforcement, it provides immediate conditioned reinforcement because onset of the positive terminal-link stimulus signals a reduction in delay to reinforcement over that signaled by the choice peck. Specific predictions regarding the effects of BOTL duration would depend upon how this general framework is delineated and, in particular, whether signaled periods of timeout are assumed to enter into determination of either the delay reduction or the rates of reinforcement associated with each alternative. If signaled periods of timeout do not enter into either the delay to reinforcement or the rate of reinforcement, then BOTL duration should be inert in signaled procedures. On the other hand, to the extent that the duration of the BOTL does affect primary and conditioned reinforcement of the choice alternatives, the effects would counteract one another. Specifically, increases in BOTL duration would reduce the rate of primary reinforcement associated with choice of the $50 \%$ alternative, but would also increase the average time to food associated with choice of the $50 \%$ alternative and thus further reduce the delay signaled by onset of the $\mathrm{S}+$.

Our third finding was that preference for the $50 \%$ side varied with the relative duration of the food terminal link on that side. In general, when the food terminal link on the $50 \%$ side was shorter than the average time to food on the $100 \%$ side, the $50 \%$ side was preferred. However, when the food terminal link on the $50 \%$ side was longer than the average time to food on the $100 \%$ side, the $100 \%$ side was preferred. This pattern was qualitatively similar but quantitatively different for signaled and unsignaled conditions. Specifically, when the duration of the terminal link leading to food was $55 \mathrm{sec}$, the $100 \%$ side was strongly preferred in both signaled and unsignaled conditions. However, when the duration of the food terminal link on the $50 \%$ side was $5 \mathrm{sec}$, the $50 \%$ side was strongly preferred when outcomes were signaled, but only a weak preference for the $50 \%$ side occurred when outcomes were unsignaled. In evaluating preference for the $50 \%$ alternative when the food terminal link was $5 \mathrm{sec}$, it is important to remember that the $100 \%$ side provided more food overall than the $50 \%$ side and provided short delays to food just as frequently as the $50 \%$ side. Thus, in signaled conditions, pigeons displayed an extreme preference for an alternative that provided a lower overall rate of primary reinforcement.
This last result can be accounted for by both Mazur's (1989, 1991) model and the local delay-reduction framework. According to Mazur's model, the value of the $50 \%$ alternative would be greater than the value of the $100 \%$ alternative when the delay to food in the presence of the $50 \% \mathrm{~S}+$ is shorter than the average delay to food in the presence of the $100 \%$ terminal-link stimulus. The blackout terminal-link stimulus under signaled conditions is not associated with food and therefore would have no valence. Consequently, the value of the $50 \%$ alternative is affected only by the duration of the food stimulus. According to the local delay-reduction analysis, onset of the stimulus associated with food after a short delay will have greater strength as a conditioned reinforcer because that stimulus signals a substantial reduction in delay relative to the delay signaled by a choice response. Moreover, although the $100 \%$ side provides more primary reinforcement overall, the equal occurrence of short delays to primary reinforcement on both alternatives should mitigate the difference in their value based on primary reinforcement. Consequently, the conditionedreinforcement value of the $50 \%$ alternative should be much greater than the value of the $100 \%$ alternative, whereas the primary-reinforcement value of the $50 \%$ alternative should be only marginally weaker than that of the $100 \%$ alternative.

The slight preference for the $50 \%$ side under the unsignaled F5/BO55 condition is not predicted by either framework without additional assumptions. Instead, one would expect indifference or slight preference for the $100 \%$ alternative according to both the hyperbolic-decay model and the local delay-reduction framework. Since choice proportions were not significantly different from .5 according to a one-sample $t$ test $[t(11)=0.77]$, the slight preference for the $50 \%$ side may not represent a serious departure from these predictions of indifference. It is also possible that the pigeons discriminated between the first $5 \mathrm{sec}$ of the $50 \%$ stimulus, which was followed by food half of the time, and the last $50 \mathrm{sec}$ of the $50 \%$ stimulus, which was never followed by food, and thereby altered the functional delay to food associated with the 50\% terminal-link stimulus (see Mazur, 1991).

In Phase 2 of Experiment 3 and in previous studies (Spetch et al., 1990), choice proportions for the 50\% alternative in signaled procedures increased substantially as the duration of all terminal links was increased. According to the local delay-reduction framework, this result reflects the diminished control by primary reinforcement (which favors the $100 \%$ alternative) as delay to reinforcement increases. This result may also be accommodated by Mazur's $(1989,1991)$ model, at least at a qualitative level, by considering that initial-link response latencies are a functional part of the delay to the reinforcer (see Mazur, 1991). Specifically, on the 100\% alternative, the delay to reinforcement would be the terminal-link duration plus the latency to make a peck on the $100 \%$ side. On the $50 \%$ alternative, the delay to reinforcement would include the duration of the $\mathrm{S}+$ terminal link, the latency to the peck that produced that 
stimulus, and the latency to pecks that produced a blackout stimulus prior to onset of the $\mathrm{S}+$. Thus, on average, two pecks with their associated latencies would be required per reinforcer on the $50 \%$ side. The contribution of initial-link latencies to the value of an alternative would decrease as terminal-link durations are increased because they would constitute a decreasing proportion of the total functional delay. Consequently, at shorter terminal-link durations, the $100 \%$ side should be preferred because the longer delay produced by the extra initiallink peck latencies would make the $50 \%$ side less valuable. As the terminal-link duration increases, the difference between the two sides produced by the extra peck latencies diminishes and preference should dissipate toward indifference.

Overall, many of the results obtained fit well with both Mazur's $(1989,1991)$ model and the local delayreduction approach proposed by Dunn and Spetch (1990). Mazur's model provides a more parsimonious account than that offered by the delay-reduction framework for the effect of varying BOTL duration in unsignaled procedures and the lack of effect of this same manipulation within signaled procedures. His model is also formalized to allow quantitative predictions, whereas the local delay-reduction framework has not yet been formally developed to allow quantitative predictions. Nevertheless, it may be premature to reject the delayreduction framework in favor of the hyperbolic-decay model because without some additional assumptions, the hyperbolic-decay model may not account for some aspects of pigeons' choice between certain and probabilistic outcomes. In particular, Mazur's model predicts that, in signaled procedures, pigeons should show either a preference for the $100 \%$ alternative or indifference between the two alternatives. Because between-subject variability is typically high, we cannot strongly argue against these predictions on the basis of the results presented here. It is noteworthy, however, that in each of the present experiments, as well as in those of Dunn and Spetch (1990), Kendall (1974, 1985), and Spetch et al. (1990), mean choice proportions for the 50\% side were above .5 in all signaled conditions with equal terminal links of $30 \mathrm{sec}$ or longer. Furthermore, in a recent study (Belke, 1992; Belke \& Spetch, 1994), 6 out of 8 pigeons displayed a strong preference for the $50 \%$ alternative in a variant of the present procedure in which they were forced to remain on the $50 \%$ side after they chose it until a food outcome occurred. Key reversal tests indicated that the strong preference for the $50 \%$ alternative was not due to a position bias, and tests in which onset of the terminal-link stimuli was delayed clearly implicated conditioned reinforcement from onset of the terminallink stimuli in this preference. Mazur's model does not predict preference for the $50 \%$ alternative, whereas the local delay-reduction framework does allow such a result. For this reason, it appears prudent to continue to explore both frameworks until evidence that strongly favors one over the other is found.
There are two main points of difference between Mazur's (1989, 1991) model and the local delay-reduction approach. First, Mazur's model combines the effect of primary reinforcement and conditioned reinforcement into a single value, whereas the local delay-reduction framework assumes that primary and conditioned reinforcement can each work separately to determine the value of an alternative. Second, Mazur's model holds that value is a function of the absolute delay to food, whereas the delay-reduction approach adds a role for local context in the conditioned-reinforcing effectiveness of a stimulus associated with food. Thus, the delayreduction approach adopts the assumption that "reinforcement is inherently relativistic" (Williams, 1988, p. 215) and that the context in which it occurs is a critical determinant of its value (see Grace, 1993).

\section{REFERENCES}

Barnet, R. C., Grahame, N. J., \& Miller, R. R. (1993). Local context and the comparator hypothesis. Animal Learning \& Behavior, 21, 1-13.

Belke, T. W. (1992). Preference for less frequent reinforcement. Poster presented at the meeting of the Association for Behavior Analysis, San Francisco, CA.

Belke, T. W., \& Spetch, M. L. (1994). Choice between reliable and unreliable alternatives revisited: Preference for unreliable reinforcement. Manuscript submitted for publication.

CARACO, T. (1983). White-crowned sparrows (Zonotrichia leucophrys): Foraging preferences in a risky environment. Behavioral Ecology \& Sociobiology, 12, 63-69.

Collins, L., \& Pearce, J. M. (1985). Predictive accuracy and the effects of partial reinforcement on serial autoshaping. Journal of Experimental Psychology: Animal Behavior Processes, 11, 548-564.

Collins, L., Young, D. B., Davies, K., \& Pearce, J. M. (1983). The influence of partial reinforcement on serial autoshaping with pigeons. Quarterly Journal of Experimental Psychology, 35B, 275290.

Dunn, R. (1990). Timeout from concurrent schedules. Journal of the Experimental Analysis of Behavior, 53, 163-174.

DunN, R., \& Spetch, M. L. (1990). Choice with uncertain outcomes: Conditioned reinforcement effects. Journal of the Experimental Analysis of Behavior, 53, 201-218.

FAntino, E. (1969). Choice and rate of reinforcement. Journal of the Experimental Analysis of Behavior, 12, 723-730.

FAntino, E., \& CASE, D. A. (1993). The delay-reduction hypothesis: Effects of informative events on response rates and choice. Quarterly Journal of Experimental Psychology, 46B, 145-161.

FARLEY, J. (1980). Automaintenance, contrast, and contingencies: Effects of local vs. overall and prior vs. impending reinforcement context. Learning \& Motivation, 11, 19-48.

Grace, R. C. (1993). Violations of transitivity: Implications for a theory of contextual choice. Journal of the Experimental Analysis of Behavior, 60, 185-201.

Herrnstein, R. J., \& Vaughan, W., Jr. (1980). Melioration and behavioral allocation. In J. E. R. Staddon (Ed.), Limits to action: The allocation of individual behavior (pp. 143-176). New York: Academic Press.

HurSh, S. R., \& FANTINO, E. (1974). An appraisal of preference for multiple versus mixed schedules. Journal of the Experimental Analysis of Behavior, 22, 31-38.

Kendall, S. B. (1974). Preference for intermittent reinforcement. Journal of the Experimental Analysis of Behavior, 21, 463-473.

Kendall, S. B. (1985). A further study of choice and percentage reinforcement. Behavioral Processes, 10, 399-413.

KilleEN, P. R. (1982). Incentive theory: II. Models for choice. Journal of the Experimental Analysis of Behavior, 38, 217-232. 
Krebs, J. R. (1978). Optimal foraging: Decision rules for predators. In J. R. Krebs \& N. B. Davies (Eds.), Behavioural ecology: An evolutionary approach (pp. 23-63). Sunderland, MA: Sinauer.

MAzUR, J. E. (1989). Theories of probabilistic reinforcement. Journal of the Experimental Analysis of Behavior, 51, 87-99.

MAZUR, J. E. (1991). Choice with probabilistic reinforcement: Effects of delay and conditioned reinforcers. Journal of the Experimental Analysis of Behavior, 55, 63-77.

Mazur, J. E. (1993). Predicting the strength of a conditioned reinforcer: Effects of delay and uncertainty. Current Directions in Psychological Science, 2, 70-74.

Mazur, J. E., \& Romano, A. (1992). Choice with delayed and probabilistic reinforcers: Effects of variability, time between trials, and conditioned reinforcers. Journal of the Experimental Analysis of Behavior, 58, 513-525.

Pearce, J. M., \& Collins, L. (1987). An evaluation of the associative strength of a partially reinforced serial CS. Quarterly Journal of Experimental Psychology, 39B, 273-293.

Pyke, G. H., Pulliam, H. R., \& Charnov, E. L. (1977). Optimal foraging: A selective review of theory and tests. Quarterly Review of Biology, 52, 137-154.

Rachlin, H., Logue, A. W., Gibbon, J., \& Frankel, M. (1986). Cognition and behavior in studies of choice. Psychological Review, 93, $33-45$.

SHIMP, C. P. (1966). Probabilistically reinforced choice behavior in pigeons. Journal of the Experimental Analysis of Behavior, 9, 443455 .

Spetch, M. L., Belke, T. W., Barnet, R. C., Dunn, R., \& Pierce, W. D. (1990). Suboptimal choice in a percentage-reinforcement procedure: Effects of signal condition and terminal- link length. Journal of the Experimental Analysis of Behavior, 53, 219-234.

Spetch, M. L., \& RusaK, B. (1989). Pigeons' memory for event duration: Intertrial interval and delay effects. Animal Learning \& Behavior, 17, 147-156.

SPETCH, M. L., \& RUSAK, B. (1992). Temporal context effects in pigeons' memory for event duration. Learning \& Motivation, 23, 117-144

Terrace, H. S., Gibbon, J., Farrell, L., \& Baldock, M. D. (1975). Temporal factors influencing the acquisition and maintenance of an autoshaped keypeck. Animal Learning \& Behavior, 3, 53-62.

Williams, B. A. (1979). Contrast, component duration, and the following schedule of reinforcement. Journal of Experimental Psychology: Animal Behavior Processes, 5, 379-396.

Williams, B. A. (1988). Reinforcement, choice, and response strength. In R. C. Atkinson, R. J. Herrnstein, G. Lindzey, \& R. D. Luce (Eds.), Stevens' handbook of experimental psychology: Vol 2. Learning and cognition (pp. 167-244). New York: Wiley.

Williams, B. A. (1992a). Competition between stimulus-reinforcer contingencies and anticipatory contrast. Journal of the Experimental Analysis of Behavior, 58, 287-302.

WiLliams, B. A. (1992b). Inverse relations between preference and contrast. Journal of the Experimental Analysis of Behavior, 58, 303312.

Williams, B. A., \& DunN, R. (1991). Preference for conditioned reinforcement. Journal of the Experimental Analysis of Behavior, 55, $37-46$.

(Manuscript received September 7, 1993; revision accepted for publication January 28, 1994.) 